\title{
Why the transitivity of perceptual simultaneity should be taken seriously
}

\section{Valtteri Arstila*}

Department of Behavioral Sciences and Philosophy, University of Turku, Turku, Finland

*Correspondence: valtteri.arstila@utu.fi

A relation is transitive if and only if from the fact that $\mathrm{A}$ has a relation to $\mathrm{B}$, and $\mathrm{B}$ has a relation $C$, it necessarily follows that $A$ also has a relation to $\mathrm{C}$. As a relation fulfilling this requirement, consider simultaneity understood as two events occurring at the same time and only at the same time (thus this is not a case of mere partial temporal overlap): If $A$ and $B$ share all the same temporal properties, and $\mathrm{B}$ and $\mathrm{C}$ share them too, then it is impossible for $\mathrm{A}$ and $\mathrm{C}$ not to share them too. Accordingly, the simultaneity of the time of events is a transitive relation. On the other hand, "to be non-simultaneous (asynchronous)" is not a transitive relation because $A$ and $C$ can be simultaneous although both of them are non-simultaneous with $\mathrm{B}$.

Perceptual simultaneity refers to our perception or judgment that two or more things are simultaneous (or at least that we cannot tell their temporal order). While the transitivity of the simultaneity of time of events is virtually always accepted, philosopher Kelly (2005) has argued that perceptual simultaneity is an intransitive relation. If successful, and the conclusions drawn from this claim were sound, Kelly's (2005) argument would pose a serious challenge to certain theories of consciousness and views on neural synchrony (Elliott et al., 2006). Instead of elaborating on these consequences, and how they follow from the possible intransitivity of perceptual simultaneity, I will argue that the argument itself rests on an assumption that requires justification. Accordingly, it should remain an open question whether perceptual simultaneity is a transitive or intransitive relation, and addressing this question would be a fruitful empirical endeavor.

Kelly's argument takes the imprecision of perceptual simultaneity as its starting point. It is indeed a well-known fact that when two stimuli are presented in a short temporal asynchrony, we judge them to be simultaneous. Depending on the nature of the stimuli, this window of simultaneity - temporal extension during which we cannot tell the temporal order of asynchronous stimuli can range from few milliseconds to tens of milliseconds. Based on this imperfection of our perceptual processes, Kelly's argument focuses on situations where subjects are shown three or more asynchronous stimuli. One way to present it goes as follows.

Let us assume that a subject is shown three brief asynchronous stimuli (A, B, and C) such that the onset asynchrony between $A$ and $B$ is three quarters of the temporal window of simultaneity, and that this holds for $\mathrm{B}$ and $\mathrm{C}$ too. Accordingly, $\mathrm{A}$ and $\mathrm{B}$, and $\mathrm{B}$ and $\mathrm{C}$ are judged to be simultaneous. But the onset asynchrony between $\mathrm{A}$ and $\mathrm{C}$ is $50 \%$ longer than the window of simultaneity. Hence, $\mathrm{A}$ and $\mathrm{C}$ are not perceptually simultaneous. Given that the relation of perceptual simultaneity holds between $A$ and $B$, and between $B$ and $C$, but not between $A$ and $C$, perceptual simultaneity turns out to be an intransitive relation.

To make this more concrete, let us assume that the temporal window of simultaneity is $40 \mathrm{~ms}$ (although the exact number has no bearing on the success of the argument, this is close the lower limit of the estimations for visual simultaneity threshold). In this case A and B, and B and C could be presented $30 \mathrm{~ms}$ apart, and they are perceptually simultaneous. Nevertheless, A and $\mathrm{C}$ appear $60 \mathrm{~ms}$ apart, which makes them perceptually asynchronous.

The previous line of reasoning rests, however, on (at least thus far unjustified) assumption because the intransitivity of perceptual simultaneity does not follow by logical necessity from the imperfection of our perceptual processes to determine temporal relations between stimuli. To see this, consider Kelly's argument again. In order for the argument to get off the ground, three comparisons need to be established: while we perceive A and B as simultaneous, we also perceive $\mathrm{B}$ and $\mathrm{C}$ as simultaneous and $\mathrm{A}$ and $\mathrm{C}$ as non-simultaneous. For this to take place, the argument needs to entail that we can successfully make a number of simultaneity comparison that cover the same episodes of experiencing. If it turned out, for example, that the episode of experiencing $\mathrm{B}$ cannot be part of two simultaneity comparisons, one for $\mathrm{A}$ and $\mathrm{B}$ and the other for B and C, then the simultaneity of one of them cannot be established and at least one of the premises of the argument for intransitivity would lose its grounding. Kelly's argument is therefore based on a hidden assumption of parallelity according to which two or more simultaneity comparisons can partly cover the same moments of experiencing. (Because the simultaneity comparisons are done based on the information still lingering in our consciousness rather than, say, based on our memory of yesterday's events, the assumption of parallelity amounts roughly to the idea that we can do more than one simultaneity comparison before the information disappears.) If one holds the assumption opposite to the one that Kelly is making - the assumption of seriality according to which simultaneity comparisons cannot cover the same moments of experiencing - then there is no argument for the intransitivity.

It thus remains an open question whether perceptual simultaneity is transitive or not. Nevertheless, in addition to the general principle that one should not adopt any assumptions - especially those that may have significant consequences without testing them, two experiments suggest that the assumption of parallelity along with the intransitivity of perceptual simultaneity may not hold. It is worth noticing that the following cases concern only visual modalities, and whether they can be extended to apply to other sensory modalities as well as to crossmodal simultaneity is an open question.

First, the issue whether the visual judgments of simultaneity are transitive or not has been explicitly tested by Corwin and Boynton (1968). Based on their results, they concluded that " $[\mathrm{w}]$ ithin the experimental 
variability obtained, the transitivity relation was confirmed" (Corwin and Boynton, 1968, p. 560). Subsequently, their study provides support for the assumption of seriality and against the assumption of parallelity.

Second, rather than testing transitivity directly, one can also wonder what happens when more than two asynchronous stimuli are presented. An indication to this can be found from a study done by Lichtenstein (1961), where subjects were presented flashes in four locations with varying asynchronies between them (and then the cycle began again). The results showed that despite large onset asynchronies (up to $95 \mathrm{~ms}$ ), all four flashes were perceived to be simultaneous. More precisely, they appeared to pulsate together in a manner that "no time difference among any of the pulsating dots is discernible" (Lichtenstein, 1961, p. 56). That is, adding more asynchronous stimuli to the comparison appeared to make the temporal window of simultaneity considerably wider than it is usually for visual stimuli. On the other hand, the pulsation of stimuli implies that after a critical period of time, one temporal window of simultaneity ends and another begins - meaning that perceptual contents are part of only one perceptual simultaneity comparison at a time as suggested by the assumption of seriality.

There are thus one explicit and one indirect reason to think that the transitivity and the assumption of seriality might be true. Such evidence is hardly conclusive, however, and therefore it remains an open question whether this is the case or not. In addition of merely settling the issue of transitivity, addressing this question would also be a fruitful way for testing the theoretical models of simultaneity judgments - something that would be lost if we simply assumed that perceptual simultaneity is transitive or intransitive. This is because the models put forward to explain our simultaneity judgments usually imply that perceptual simultaneity is either a transitive or an intransitive relation. Let me finish with examples of this.

When thinking about perceptual simultaneity, some psychologists have postulated an existence of a comparator that compares the arrival times of sensory messages produced by presented stimuli. It has a temporal resolution, a limit below which it cannot separate the temporal order of the stimuli and if the difference of sensory messages produced by some stimuli is below this threshold, the comparator regards them as simultaneous. It is worth noticing that the idea of comparator can be either understood literally (Efron, 1963) or merely as a useful theoretical construct to account our performance in simultaneity judgment tasks (Ulrich, 1987). Thus, even if there is no real simultaneity-comparator in the brain, the following models can be understood as providing different theoretical accounts of the key factors influencing how we come about judging that two stimuli are simultaneous.

One example of how this comparator could function originates from von Baer (1862) and was later revived by Stroud (1955). According to this discrete moment hypothesis, one temporal window of simultaneity (one perceptual moment) follows another in discrete fashion - when one temporal window ends, another one begins immediately. Everything that occurs (or is registered) within one perceptual moment is considered simultaneous, while everything outside of this perceptual moment is non-simultaneous. Because "belonging to a single perceptual moment" determines the perceptual simultaneity, and this relation is transitive, on this model perceptual simultaneity is also a transitive relation. The same also applies to the triggered moment hypothesis that resembles Stroud's proposal with the exception that here the comparator begins to do its task only when it has been triggered by a stimulus - temporal windows of simultaneity do not follow each other automatically in the absence of stimuli. Lichtenstein's (1961) results, in turn, suggest that the window of simultaneity is partly determined by the properties of the stimuli. Accordingly, they imply a model that is likely to be a version of the triggered model hypothesis. Unlike in other models discussed here though, the window of simultaneity is flexible. Thus it could be called triggered flexible moment hypothesis. Nevertheless, as mentioned above, here the perceptual simultaneity is a transitive relation. Allport (1968) traveling moment hypothesis, on the other hand, maintains that there is only one continuously moving perceptual moment. Because the perceptual moment moves continuously, two different perceptual moments can partly cover the same episode of experiencing in Allport's model. Consequently, this model is compatible with the assumption of parallelity and if it were correct, then perceptual simultaneity would turn out to be an intransitive relation. At this point it remains an open question which one of these models, if any, explain the empirical data best. For this reason, the issue of transitivity of perceptual simultaneity should be taken seriously because it would provide an additional way to separate these and similar models.

To sum up, all means of "measuring" are somewhat imprecise and our ability to determine asynchronies is not an exception. Accordingly, we can never know whether two events occurred exactly at the same time or not. Nevertheless, the intransitivity of perceptual simultaneity does not follow by logical necessity from this imperfection because it requires further that the assumption of parallelity is true - that two or more simultaneity comparisons can (partly) cover the same moments of experiencing. Moreover, the two discussed empirical studies bearing relevance on the matter suggest that that perceptual simultaneity may not be a transitive relation after all and thus that the assumption of parallelity is not true. On the other hand, as this evidence is not conclusive, conducting more research on the topic would be valuable.

\section{ACKNOWLEDGMENTS}

I am very grateful for Dr. Christine Falter for the countless stimulating discussions on the topic. I also want to thank the participants of "Neurobiology of Time Perception: from normality to dysfunction" satellite meeting at EBBS on Seville for their constructive and helpful comments. This work was supported by the Academy of Finland and a European Cooperation in Science and Technology (COST) action on Time in Mental Activity (TIMELY; TD0904).

\section{REFERENCES}

Allport, D. A. (1968). Phenomenal simultaneity and the perceptual moment hypothesis. Br. J. Psychol. 59, 395-406.

Corwin, T. R., and Boynton, R. M. (1968).Transitivity of visual judgments of simultaneity. J. Exp. Psychol. $78,560-568$.

Efron, R. (1963). The effect of handedness on the perception of simultaneity and temporal order. Brain 86, 261-284.

Elliott, M.A., Shi, Z., and Kelly, S. D. (2006). A moment to reflect upon perceptual synchrony. J. Cogn. Neurosci. 18, 1663-1665.

Kelly, S.D. (2005), "The puzzle of temporal experience," in Cognition and the Brain: The Philosophy 
and Neuroscience Movement, eds A. Brook and K. Akins (Cambridge: Cambridge University Press), 218-248.

Lichtenstein, M. (1961). Phenomenal simultaneity with irregular timing of components of the visual stimulus. Percept. Mot. Skills 12, 47-60.

Stroud, J. (1955). "The fine structure of psychological time," in Information Theory in Psychology, Vol. 138, ed. H. Quastler (Glencoe, IL: Free Press), 623-631.
Ulrich, R. (1987). Threshold models of temporalorder judgments evaluated by a ternary response task. Percept. Psychophys. 42, 224-239.

von Baer, K. E. (1862). Welche Auffassung der lebenden Natur ist die richtige? und Wie ist diese Auffassung auf die Entomologie anzuwenden? Berlin: A. Hirschwald.

Received: 18 October 2011; accepted: 10 January 2012; published online: 31 January 2012.
Citation: Arstila V (2012) Why the transitivity of perceptual simultaneity should be taken seriously. Front. Integr. Neurosci. 6:3. doi: 10.3389/fnint.2012.00003

Copyright $\odot 2012$ Arstila. This is an open-access article distributed under the terms of the Creative Commons Attribution Non Commercial License, which permits non-commercial use, distribution, and reproduction in other forums, provided the original authors and source are credited. 